\title{
Associations of BRAF V600E, clinical pathology and imaging factors with the recurrence rate of papillary thyroid microcarcinoma
}

\author{
KUN HUANG $^{1}$, NINGNING GAO ${ }^{2}$, DONGLIN BIAN ${ }^{1}$, QIXI ZHAI $^{1}$, PUXU YANG $^{1}$ and YUNFEI ZHANG ${ }^{1}$ \\ ${ }^{1}$ Department of Ultrasonic Diagnosis, The First Affiliated Hospital of China Medical University, Shenyang, Liaoning 110001; \\ ${ }^{2}$ Department of Ultrasonic Diagnosis, Liaoning Province Cancer Hospital and Institute, Shenyang, Liaoning 110042, P.R. China
}

Received September 13, 2019; Accepted May 13, 2020

DOI: $10.3892 / \mathrm{etm} .2020 .9373$

\begin{abstract}
In the present study, the recurrence rate of papillary thyroid microcarcinoma (PTMC) was assessed by analyzing postoperative follow-up data of affected patients and its associations with BRAF V600E, clinical pathology and imaging factors were explored. A total of 506 patients with PTMC were selected who underwent surgery from January 2014 to March 2016. The maximal diameter of thyroid nodules was $\leq 1 \mathrm{~cm}$ and all patients who underwent BRAF V600E testing and evaluation for lymph node metastasis. Postoperatively, each patient was regularly followed up to detect recurrence. Categorical variables were comparatively analyzed using univariate Cox linear regression analysis to screen for protective and adverse factors influencing recurrence of PTMC. A stepwise Cox proportional hazards regression model analysis was performed to explore risk factors affecting recurrence. Among the 506 patients, 477 were followed up, 29 were lost to follow-up and 26 patients experienced recurrence. The 5-year recurrent rate of PTMC was $5.45 \%$. The univariate Cox regression analysis indicated that PTMC recurrence was influenced by BRAF V600E, sex, multifocality, capsular invasion and lateral cervical lymph node metastasis $(\mathrm{P}<0.05)$, but not by age, tumor location on the thyroid, size, single central lymph node metastasis, distant metastasis and operative approach $(\mathrm{P}>0.05)$. The significant factors associated with recurrent PTMC were subjected to stepwise multivariate Cox proportional hazards regression model analysis and the results indicated that BRAF V600E, sex, multifocality and lateral cervical lymph node metastasis were independent factors influencing recurrence in patients with PTMC, with a statistically significant difference $(\mathrm{P}<0.05)$. In conclusion, BRAF V600E, sex, multifocality and
\end{abstract}

Correspondence to: Dr Yunfei Zhang, Department of Ultrasonic Diagnosis, The First Affiliated Hospital of China Medical University, 155 Nanjingbei Street, Shenyang, Liaoning 110001, P.R. China E-mail: zhangyunfei0927@163.com

Key words: BRAF V600E, papillary thyroid microcarcinoma, recurrence rate, color Doppler ultrasonography lateral cervical lymph node metastasis are independent risk factors for recurrent PTMC.

\section{Introduction}

Thyroid carcinoma is the most common type of malignant tumor of the endocrine system; in recent years, there has been a gradual rise in associated morbidity and this has been increasingly studied by clinicians and researchers (1), and exhibits the fastest increase in incidence among all types of malignant tumors. The soaring rate of thyroid carcinoma is primarily due to papillary carcinoma, particularly in its earliest stages (i.e. microcarcinoma) (2). According to the latest Surveillance, Epidemiology and End Results data, nearly $90 \%$ of thyroid carcinomas are of the papillary type (3), indicating the particular importance of studying thyroid papillary microcarcinoma (PTMC). In addition, PTMC has a cervical lymph-node metastasis rate that is as high as $30-70 \%$ (4-6). Certain patients experience early postsurgical local recurrences or even initially present with distant metastases to the lungs or bone $(4,5)$.

The BRAF V600E mutation is the most common mutation identified in papillary thyroid carcinoma (PTC) (6), corresponding to $28-83 \%$ of all gene mutations observed and accounting for $\sim 90 \%$ of all BRAF mutations (7-9), but it has not been detected in normal thyroid tissues or benign lesions (10-14). The presence of the BRAF V600E mutation is closely associated with papillary carcinoma recurrence and patient mortality. In addition, numerous patients choose surgical treatment for PTMC due to the high morbidity rate (15) However, certain patients develop recurrent PTMC postoperatively (16). This study focused on BRAF V600E, clinical pathology and imaging factors to explore their associations with PTMC recurrence. According to the definition by the World Health Organization, PTC with a maximal diameter of tumor $\leq 1 \mathrm{~cm}$ is considered PTMC (17).

\section{Patients and methods}

Research subjects. The independent ethics committee of China Medical University (Shenyang, China) approved this study. In total, 506 patients were included who underwent 
surgery for a thyroid nodule (maximal diameter $\leq 1.0 \mathrm{~cm}$ ) and underwent preoperative BRAF V600E testing at the Department of Thyroid Surgery of the First Affiliated Hospital of China Medical University between January 2014 and March 2016. The cohort comprised 420 females and 86 males with an age range of 17-73 years (mean age, 39.6 \pm 2.6 years). All patients were pathologically diagnosed with PTMC, including 359 patients diagnosed with a single nodule and 147 patients with two and more nodules. The mean and median diameters of nodules were $0.63 \mathrm{~cm}$ [standard deviation (SD), $0.21 \mathrm{~cm}$ ] and $0.6 \mathrm{~cm}$ (range, $0.41-1.00 \mathrm{~cm}$ ). Partial thyroidectomy was performed for unilateral nodules and total thyroidectomy for bilateral nodules. None of the patients included had any contraindications for surgery and signed informed consent forms. Patients with a history of other tumors were excluded.

Procedures. Phillip iu22 and EPIQ7 color Doppler ultrasonic (US) diagnosis systems (Philips Medical Systems, Inc.) with a probe frequency of 5-12 $\mathrm{MHz}$ were used. Patients were placed in a supine position. The thyroid glands were scanned preoperatively to determine the size, number, position, border, shape and internal echo of thyroid nodules and the presence or absence of lymph node metastasis. Surgical treatment was performed after the diagnosis was confirmed by cytopathological evaluation. Prior to surgical resection of the thyroid nodules, BRAF V600E testing was performed using the Amplification Refractory Mutation System method with the human BRAF gene V600E mutation detection kit (Shanghai Yuanqi Biological Co., Ltd.) and the manufacturer's protocol was strictly followed. During the test, $5 \mu \mathrm{l}$ of the standardized DNA template was pipetted into $35 \mu \mathrm{l}$ of the reaction mixture containing Taq enzyme ( $40 \mu \mathrm{l}$ of the PCR reaction system). Nodule positions were classified as being in the central area or marginal area; the marginal area included anterior, posterior, lateral and medial borders and the isthmus of the thyroid gland and the central area consisted of the remaining parts of the thyroid gland.

Follow-up. All patients underwent thyroid-stimulating hormone (TSH)-suppressive therapy with levothyroxine postoperatively. At 3, 6, 12, 18, 24, 36, 48 and 60 months after the operation, each patient was followed up, and US scan, thyroglobulin (TG) and thyroid function tests (including serum TSH, FT4, FT3, and anti-TG antibody) were performed to detect recurrence and metastasis. For patients with suspected or proven elevation of TG, enhanced CT and cytopathological examinations were performed to evaluate metastasis, and emission computed tomography (ECT) or positron emission tomography-CT (PET-CT) examination was performed as necessary.

To assess serum TG levels, electrochemiluminescence immunoassay using the Cobas e601 immunoassay analyzer (Roche Diagnostics) was used. Functional sensitivity is defined as the lowest analyte concentration that can be reproducibly measured with a precision coefficient of variation (CV) of $20 \%$; the functional sensitivity was $0.1 \mathrm{ng}$ per milliliter. Using a chemiluminescence immunoassay with an automated immunoassay analyzer (Architect i2000; Abbott Laboratories; reference range, 0.35-4.94 $\mu \mathrm{IU} / \mathrm{ml}$; functional sensitivity, $0.01 \mu \mathrm{IU} / \mathrm{ml}$ ), serum thyroid-stimulating hormone (TSH) levels were measured.
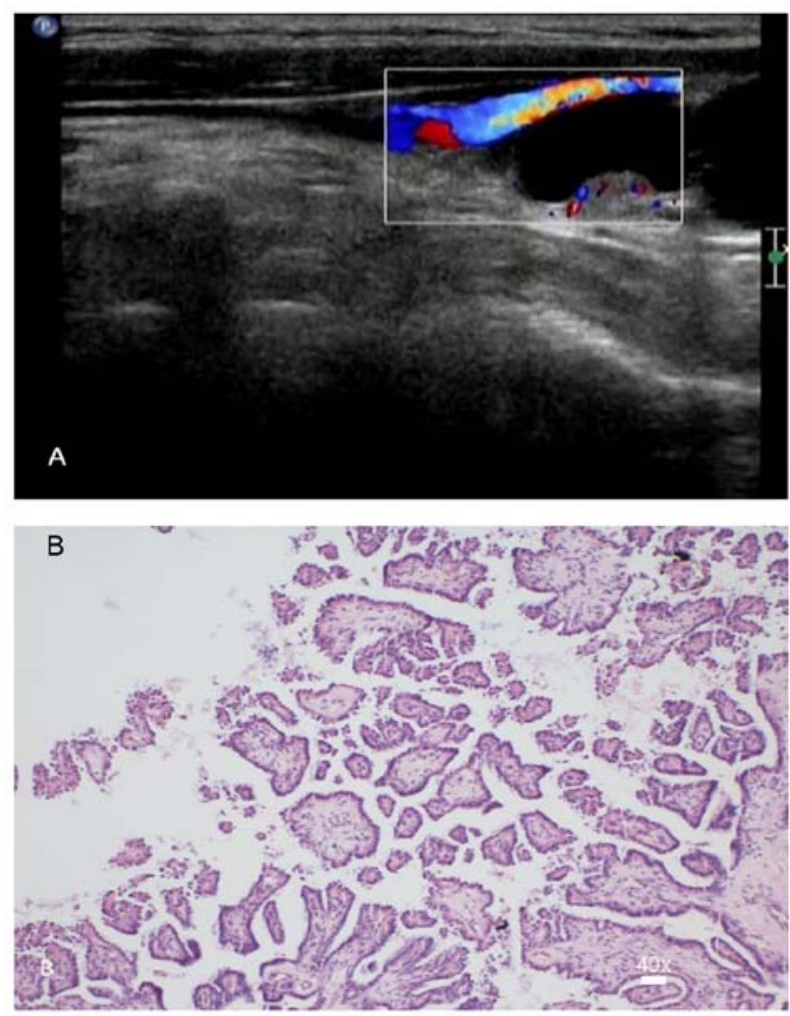

Figure 1. Female, 43 years old, recurrence 36 months after surgery. Structural recurrence of papillary thyroid microcarcinoma in a lymph node (A) Ultrasound indicates that the lymph node is cystic. (B) Pathological examination reveals cancer cells in the lymph node (magnification, x100).
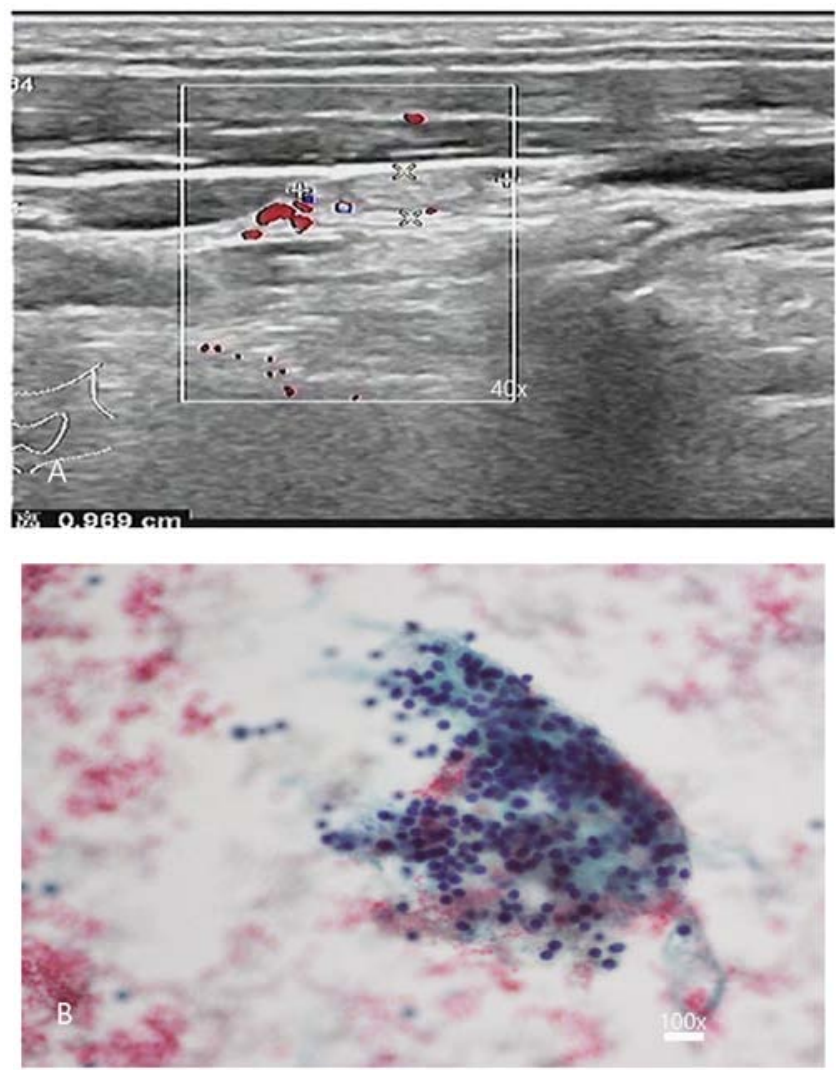

Figure 2. Female, 36 years old, appeared 24 months after surgery. Lymph node structural persistence. (A) Ultrasound indicates the loss of fatty hilum of the lymph node. (B) Cytopathological examination reveals lymphocytes and no malignant cells are observed (magnification, $\mathrm{x} 200$ ). 
Table I. The recurrence time and number of cases of papillary thyroid microcarcinoma.

\begin{tabular}{lllllllllr}
\hline Recurrence time (month) & 3 & 6 & 12 & 18 & 24 & 36 & 48 & 60 & Total \\
\hline $\mathrm{N}$ & 0 & 0 & 1 & 1 & 3 & 5 & 8 & 8 & 26 \\
\hline
\end{tabular}

A lymph node is defined as abnormal on ultrasonography if it meets any of the following criteria (12): i) Loss of fatty hilum, ii) diffuse or focal hyperechogenicity, iii) cystic changes, iv) microcalcifications, v) round shape, or vi) disorganized or peripheral vascularity. Any lymph nodes classified as abnormal underwent cytopathological examination; those in which cancer cells were detected were considered metastatic.

Recurrence. It was determined that there was no clinical evidence of disease if the following criteria were satisfied: i) Suppressed thyroglobulin (TG) $<1 \mathrm{ng} / \mathrm{ml}$, ii) stimulated TG $<2 \mathrm{ng} / \mathrm{ml}$, iii) no detectable anti-TG antibody, or iv) no evidence of disease on ultrasonography, computed tomography (CT), MRI, positron emission tomography-CT (PET-CT) or ECT at the final follow-up. Persistence was defined as suppressed $\mathrm{TG} \geq 1 \mathrm{ng} / \mathrm{ml}$, stimulated $\mathrm{TG} \geq 2 \mathrm{ng} / \mathrm{ml}$, or any evidence of disease on ultrasonography, CT, MRI, PET-CT or ECT after initial surgery and remnant ablation therapy with radioiodine. Recurrence was defined as malignancy diagnosed based on cytology results, elevated serum TG, or PET-CT, ECT in patients with no clinical evidence of disease (18). For a suspected recurrent mass detected on ultrasound, ultrasound-guided fine-needle aspiration (FNA) was usually performed. Cytopathological results were then obtained from the samples taken from the FNA and surgical excision.

Statistical analysis. SPSS v21.0 software (IBM Corp.) was used for data analysis. Measurement data are presented as the mean \pm SD and numerical data are expressed as $n(\%)$. The categorical variables associated with the recurrent rate were compared among different groups using univariate Cox linear regression analysis to screen for protective and adverse factors influencing recurrence of PTMC. Survival rates were calculated using the Kaplan-Meier method and compared using a log-rank test. A multivariate Cox proportional hazard regression model was used to estimate the mortality risk. Subsequently, stepwise multivariate co-variant analysis of the recurrence rates in different groups was performed using the Cox proportional hazards regression model to explore risk factors of recurrence. $\mathrm{P}<0.05$ was considered to indicate statistical significance.

\section{Results}

Follow-up findings. During the study, there was no case of death; 477 patients were followed up, 29 patients were lost to follow-up and 26 patients experienced recurrence; the 5-year recurrent rate was $5.45 \%$. The follow-up time from initial treatment to final clinical follow-up was 49.7 months (SD, 11.6 months; median, 48.5 months; range, 36.0-60.0 months). During follow-up, there was no case of recurrence at 3 and 6 months, 1 case of recurrence each at 12 and 18 months, 3 new
Table II. The follow-up of lymph node structural persistence.

Types of follow-up

Lymph

node structural persistence

No recurrence after surgery $(\mathrm{N})$

2

No change without undergoing surgery $(\mathrm{N})$

4

Total

6

cases of recurrence at 24 months, 5 new cases of recurrence at 36 months and 8 new cases of recurrence at 48 and 60 months (Table I).

Recurrenceparameters.Cases of structural recurrence accounted for $5.45 \%$ (26/477) and all were lymph node metastasis without secondary thyroid nodule or distant metastasis (Fig. 1); $1.26 \%$ (6/477) of cases exhibited structural persistence (Fig. 2) and biochemical recurrence accounted for $2.73 \%$ (13/477). Among the 26 patients with structural recurrence, 13 and 13 cases presented with increased serum TG levels and normal serum TG levels, respectively; all of these patients underwent surgery, including 2 cases (increased TG) who underwent surgery for secondary recurrence (The patient relapsed after undergoing a second operation following the first recurrence) and were not indicated to have recurrence at the second follow-up (Follow-up after the second operation). Out of the 6 patients with structural persistence, 2 cases underwent surgery without apparent metastasis and recurrence at the last visit, and 4 cases were observed and determined to be without significant enlargement of lymph nodes at the last follow-up (Table II).

Influencing factors of recurrence. Table III presents details of the patients with PTMC, including the presence of BRAF V600E, clinical pathology and imaging factors (e.g., sex, age, lesion location, size and number, capsular invasion, metastasis location, distant metastasis and operative approach). Univariate Cox regression analysis indicated that recurrence of PTMC was affected by BRAF V600E, sex, multifocality, capsular invasion and lateral cervical lymph node metastasis $(\mathrm{P}<0.05)$, but not by age, lesion location, size, single central lymph node metastasis, distant metastasis and operative approach $(\mathrm{P}>0.05$; Figs. S1 and S2).

As indicated by the stepwise multivariate Cox proportional hazards regression model analysis of the significant influencing factors for recurrent PTMC, sex, BRAF V600E, multifocality and lateral cervical lymph node metastasis were independent influencing factors for recurrence in patients with PTMC, with a statistically significant difference $(\mathrm{P}<0.05$; Table IV). 
Table III. Analysis of the association of BRAF V600E, clinical pathology and imaging factors with recurrence of papillary thyroid microcarcinoma.

\begin{tabular}{|c|c|c|c|c|c|}
\hline \multirow[b]{2}{*}{ Factor } & \multirow[b]{2}{*}{ Cases (n) } & \multicolumn{2}{|c|}{ Recurrence, $\mathrm{n}(\%)$} & \multirow[b]{2}{*}{$\mathrm{HR}(95 \% \mathrm{CI})$} & \multirow[b]{2}{*}{ P-value } \\
\hline & & Positive & Negative & & \\
\hline Location & & & & $0.451(0.055-3.718)$ & 0.460 \\
\hline Marginal region or near isthmus & 286 & $16(5.59)$ & $270(94.41)$ & & \\
\hline Central region & 191 & $10(5.24)$ & $181(94.76)$ & & \\
\hline Sex & & & & $1.689(1.045-3.262)$ & 0.004 \\
\hline Female & 397 & $18(4.53)$ & $379(95.47)$ & & \\
\hline Male & 80 & $8(10.00)$ & $72(90.00)$ & & \\
\hline Age (years) & & & & $1.065(0.110-10.299)$ & 0.956 \\
\hline$\geq 45$ & 204 & $14(6.86)$ & $190(93.14)$ & & \\
\hline$<45$ & 273 & $12(4.40)$ & $261(95.60)$ & & \\
\hline Anteroposterior nodule size $(\mathrm{cm})$ & & & & $0.444(0.058-3.402)$ & 0.435 \\
\hline $0.9-1.0$ & 195 & $15(7.69)$ & $180(92.31)$ & & \\
\hline$<0.9$ & 282 & $11(3.90)$ & $271(96.10)$ & & \\
\hline Nodule number & & & & $2.462(1.192-5.086)$ & 0.015 \\
\hline Single & 338 & $12(3.55)$ & $326(96.45)$ & & \\
\hline Multifocal & 139 & $14(10.07)$ & $125(89.93)$ & & \\
\hline BRAF V600E testing & & & & $2.312(1.254-4.074)$ & 0.022 \\
\hline Positive & 222 & $17(7.66)$ & $205(92.34)$ & & \\
\hline Negative & 255 & $9(3.53)$ & $246(96.47)$ & & \\
\hline Capsular invasion & & & & $1.244(1.013-2.115)$ & 0.043 \\
\hline Yes & 77 & $7(9.09)$ & $70(90.91)$ & & \\
\hline No & 400 & $19(4.75)$ & $381(95.25)$ & & \\
\hline Single central lymph node metastasis & & & & $0.991(0.863-1.137)$ & 0.894 \\
\hline Yes & 185 & $14(7.57)$ & $171(92.43)$ & & \\
\hline No & 292 & $12(4.11)$ & $280(95.89)$ & & \\
\hline Lateral cervical lymph node metastasis & & & & $1.899(1.308-2.966)$ & 0.001 \\
\hline Yes & 72 & $9(12.50)$ & $63(87.50)$ & & \\
\hline No & 405 & $17(4.20)$ & $88(95.80)$ & & \\
\hline Distant metastasis & & & & $0.151(0.009-2.529)$ & 0.189 \\
\hline Yes & 0 & $0(0)$ & $0(0)$ & & \\
\hline No & 477 & $26(5.45)$ & $451(94.55)$ & & \\
\hline Operative approach & & & & $1.012(0.954-1.074)$ & 0.682 \\
\hline Total thyroidectomy & 153 & $12(7.84)$ & $141(92.16)$ & & \\
\hline Unilateral thyroidectomy & 324 & $14(4.32)$ & $310(95.68)$ & & \\
\hline
\end{tabular}

HR, hazard ratio.

Table IV. Multivariate stepwise Cox proportional hazards regression model analysis of factors influencing the recurrence of papillary thyroid microcarcinoma.

\begin{tabular}{llr}
\hline Factor & HR (95\% CI) & P-value \\
\hline Sex (female vs. male) & $2.340(1.980,5.585)$ & 0.032 \\
BRAF V600E testing & $2.267(1.311,4.193)$ & 0.027 \\
Multifocality (yes vs. no) & $3.043(1.370,6.759)$ & 0.019 \\
Capsular invasion (yes vs. no) & $1.391(0.702-3.906)$ & 0.504 \\
Lateral cervical lymph node metastasis (yes vs. no) & $3.216(2.393,6.634)$ & 0.009
\end{tabular}

HR, hazard ratio; CI, confidence interval. 


\section{Discussion}

In recent years, the frequency of PTMC has rapidly risen and its morbidity has markedly increased (16). Although the requirement for surgery in the treatment of PTMC is debated, surgical management of nodal metastasis of PTMC is undisputed and the rate of nodal metastasis is high (16). For instance, the reported rates of central lymph node metastasis alone range from 24 to $64 \%$ and its occurrence is associated with recurrence and death rates $(19,20)$. However, there is no expert consensus on the susceptibility of PTMC types to recurrence. In the present study, the risk of PTMC recurrence with respect to BRAF V600E, clinical pathology factors and imaging factors was analyzed; this analysis provided a detailed assessment of the biological behavior and relapse rate associated with the BRAF mutation status.

Various studies have completed multi-year postoperative follow-ups of patients with PTMC, but they have drawn different conclusions. Hay et al (21) reported on 900 patients with PTMC diagnosed and treated at the Mayo Clinic from 1945 to 2004, with an average follow-up duration of 17.2 years; the 20-year and 40-year tumor recurrence rates were 6 and $8 \%$, respectively. Out of 281 patients with PTMC treated at the Gustave-Roussy Institute, 3.9\% of cases experienced local recurrence and 1 case developed pulmonary metastasis (22). In a study on patients with low-risk thyroid carcinoma (stage I or II), the recurrence rate was 8.9\% (23-28). However, Nixon et al (29) reported that PTMC had a low recurrence rate of only $0.6 \%$. Durante et al (30), confirmed that none of their 312 patients with PTMC T1NOM0 experienced local recurrence during a median 6-year follow-up. These conflicting results may be due to different stages of primary carcinoma, different FNA diagnostic criteria and a difference in the size criterion of US-FNA used for suspected cervical lymph node lesions. The 5-year recurrence rate in the present study was $5.45 \%$, which is similar to the 20-year follow-up results of the study by Hay et al (21) and close to those reported by the Gustave-Roussy Institute (22). However, the recurrence rate would certainly increase with the prolongation of follow-up. Despite slow growth, thyroid carcinoma has exhibited a high incidence rate in recent years. Furthermore, as tumor heterogeneity is observed, the importance of biological behaviors of PTMC should be recognized.

The univariate analysis using univariate Cox regression analysis indicated that BRAF V600E, sex, multifocality, capsular invasion and lateral cervical lymph node metastasis affected the recurrent rate of PTMC, according to the stepwise multivariate Cox proportional hazards regression model analysis, BRAF V600E, sex, multifocality and lateral cervical lymph node metastasis were independent influencing factors for recurrence in patients with PTMC (HR=2.267, 2.340, 3.043 and 3.216, respectively), which was consistent with the results of several previous studies (31-33). Li et al (34), reported a close association of BRAF V600E mutation with extracapsular infiltration of PTC, lymph node metastasis and high TNM tumor stage, and a subsequent higher capsular invasion rate (35). BRAF V600E-variant PTC has a relatively higher risk of invasion, easily invades the tissues surrounding the thyroid gland, and means that patients frequently present at an advanced clinical stage $(31,36,37)$ and is associated with a worse prognosis $(36,38,39)$. Therefore, for patients with BRAF V600E-positive tumors, more attention should be paid to US reexamination and TG and thyroid function tests in order to avoid markedly elevated TSH levels. In addition, surgery should be performed as soon as possible when BRAF V600E-positive nodes are detected.

Sex, multifocality and lateral cervical lymph node metastasis, as independent clinical pathology and imaging factors influencing PTMC recurrence, are mainly associated with the characteristics of PTMC. The incidence rate of PTMC in females is markedly higher than that in males (up to 3.9-fold) (40), but the recurrence rate exhibits the opposite trend, being higher in males $(10.00 \%)$ than in females $(4.53 \%)$. However, a larger sample size is required to further validate the accuracy of results because of the small number of cases with recurrence. Multifocality, as an independent risk factor for recurrence, contributes to the high morbidity (up to 25.7\%) of PTMC and is associated with BRAF V600E mutation (32). Therefore, regular US and TG monitoring are required for patients with multifocality. Wada et al (33), indicated that the recurrence rate of patients with lateral cervical lymph node metastasis was higher than that of patients with only central lymph node metastasis and the difference was statistically significant; this is comparable to the present results. The major cause for this is that central lymph nodes are located in the VI area, the first location of thyroid carcinoma metastasis, while lateral cervical lymph nodes are located at the II, III and IV areas and are usually the second location of metastasis; the lymph drainage generally reaches the second location in the middle and advanced tumor stages. In addition, patients with lateral cervical lymph node metastasis are frequently at least stage T1N1bM0, and they are more susceptible to recurrence and metastasis than patients with stage T1N1aM0. In addition, the cutoff criterion of the thyroid nodule size in the present study was $0.9 \mathrm{~cm}$, mainly because if the nodule size is $>9 \mathrm{~mm}$, its risk of metastasis increases, compared with it risk decreasing if the size is $<9 \mathrm{~mm}$ (41).

The present study has the following limitations. First, it was a retrospective analysis and the exclusion of certain cases was unavoidable. Furthermore, the sample size was relatively small and a larger sample size is required. In addition, the follow-up time was short and requires to be prolonged to further validate the conclusion of the present study. In addition, the imaging characteristics of nodules were not considered, which will be investigated in future studies by our group.

In conclusion, PTMC has a low recurrence rate after surgery; BRAF V600E, sex, multifocality and lateral cervical lymph node metastasis are independent risk factors for recurrent PTMC.

\section{Acknowledgements}

Not applicable.

\section{Funding}

No funding was received.

\section{Availability of data and materials}

All data generated or analyzed during this study are included in this published article. 


\section{Authors' contributions}

$\mathrm{KH}$ conceived this article, revised it critically for important intellectual content and was responsible for the interpretation of data. NG and PY made substantial contributions to acquisition and analysis of data. DB and QZ constructed the figures and involved in the acquisition of data. YZ collected the data regarding the patients, drafted the manuscript and approved it for publication. The final version of the manuscript has been read and approved by all authors, and each author believes that the manuscript represents honest work.

\section{Ethics approval and consent to participate}

The present study was approved by the Ethics Committee of the First Affiliated Hospital of China Medical University (Shenyang, Liaoning, China; no. AF-SOP-07-1.0-01).

\section{Patient consent for publication}

Not applicable.

\section{Competing interests}

The authors declare that they have no competing interests.

\section{References}

1. Huang K, Bai Z, Bian D, Yang P, Li X and Liu Y: Diagnostic accuracy of contrast-enhanced ultrasonography in papillary thyroid microcarcinoma stratified by size. Ultrasound Med Biol 46: 269-274, 2020.

2. Chen AY, Jemal A and Ward EM: Increasing incidence of differentiated thyroid cancer in the United States, 1988-2005. Cancer 115: 3801-3807, 2009.

3. Davies L and Welch HG: Increasing incidence of thyroid cancer in the United States, 1973-2002. JAMA 295: 2164-2167, 2006.

4. Xu YH, Song HJ, Qiu ZL and Luo QY: Brain metastases with exceptional features from papillary thyroid carcinoma: Report of three cases. Hell J Nucl Med 14: 56-59, 2011.

5. Varsavsky M, Cortés Berdonces M, Alonso G, García Martín A and Muñoz Torres M: Metastatic adenopathy from a thyroid microcarcinoma: Final diagnosis of a presumed paraganglioma. Endocrinol Nutr 58: 143-144, 2011 (In Spanish).

6. Xing M, Westra WH, Tufano RP, Cohen Y, Rosenbaum E, Rhoden KJ, Carson KA, Vasko V, Larin A, Tallini G, et al: BRAF mutation predicts a poorer clinical prognosis for papillary thyroid cancer. J Clin Endocrinol Metab 90: 6373-6379, 2005.

7. Kim SW, Lee JI, Kim JW, Ki CS, Oh YL, Choi YL, Shin JH, Kim HK, Jang HW and Chung JH: BRAFV600E mutation analysis in fine-needle aspiration cytology specimens for evaluation of thyroid nodule: A large series in a BRAFV600E-prevalent population. J Clin Endocrinol Metab 95: 3693-3700, 2010.

8. Min HS, Lee C and Jung KC: Correlation of immunohistochemical markers and BRAF mutation status with histological variants of papillary thyroid carcinoma in the Korean population. J Korean Med Sci 28: 534-541, 2013.

9. Kwak JY, Kim EK, Chung WY, Moon HJ, Kim MJ and Choi JR: Association of BRAFV600E mutation with poor clinical prognostic factors and US features in Korean patients with papillary thyroid microcarcinoma. Radiology 253: 854-860, 2009.

10. Millington GW: Mutations of the BRAF gene in human cancer, by Davies et al. (Nature 417: 949-954, 2002). Clin Exp Dermatol 38: 222-223, 2013.

11. DeLuca AM, Srinivas A and Alani RM: BRAF kinase in melanoma development and progression. Expert Rev Mol Med 10: e6, 2008.

12. Liu Z, Lv T, Xie C and Di Z: BRAF V600E gene mutation is associated with bilateral malignancy of papillary thyroid cancer. Am J Med Sci 356: 130-134, 2018.
13. Kebebew E, Weng J, Bauer J, Ranvier G, Clark OH, Duh QY, Shibru D, Bastian B and Griffin A: The prevalence and prognostic value of BRAF mutation in thyroid cancer. Ann Surg 246: 466-471, 2007

14. Kim TH, Park YJ, Lim JA, Ahn HY, Lee EK, Lee YJ, Kim KW, Hahn SK, Youn YK, Kim KH, et al: The association of the BRAF(V600E) mutation with prognostic factors and poor clinical outcome in papillary thyroid cancer: A meta-analysis. Cancer 118: 1764-1773, 2012

15. Gschwandtner E, Klatte T, Swietek N, Bures C, Kober F, Ott J, Schultheis A, Neuhold $\mathrm{N}$ and Hermann M: Increase of papillary thyroid microcarcinoma and a plea for restrictive treatment: A retrospective study of 1,391 prospective documented patients. Surgery 159: 503-511, 2016.

16. Yi D, Song P, Huang T, Tang X and Sang J: A meta-analysis on the effect of operation modes on the recurrence of papillary thyroid microcarcinoma. Oncotarget 8: 7148-7156, 2017.

17. Sobin LH: Histological typing of thyroid tumours. Histopathology 16: 513, 1990.

18. Yoon JH, Lee HS, Kim EK, Youk JH, Kim HG, Moon HJ and Kwak JY: Short-term follow-up us leads to higher false-positive results without detection of structural recurrences in PTMC. Medicine (Baltimore) 95: e2435, 2016.

19. Huang XP, Ye TT, Zhang L, Liu RF, Lai XJ, Wang L, Yang M, Zhang B, Li XY, Liu ZW, et al: Sonographic features of papillary thyroid microcarcinoma predicting high-volume central neck lymph node metastasis. Surg Oncol 27: 172-176, 2018.

20. Ferris RL, Baloch Z, Bernet V, Chen A, Fahey TJ III, Ganly I, Hodak SP, Kebebew E, Patel KN, Shaha A, et al: American thyroid association statement on surgical application of molecular profiling for thyroid nodules: Current impact on perioperative decision making. Thyroid 25: 760-768, 2015.

21. Hay ID, Hutchinson ME, Gonzalez-Losada T, McIver B, Reinalda ME, Grant CS, Thompson GB, Sebo TJ and Goellner JR: Papillary thyroid microcarcinoma: A study of 900 cases observed in a 60-year period. Surgery 144: 980-988, 2008.

22. Baudin E, Travagli JP, Ropers J, Mancusi F, Bruno-Bossio G, Caillou B, Cailleux AF, Lumbroso JD, Parmentier C and Schlumberger M: Microcarcinoma of the thyroid gland: The Gustave-Roussy Institute experience. Cancer 83: 553-559, 1998.

23. Pacini F, Molinaro E, Castagna MG, Agate L, Elisei R, Ceccarelli C, Lippi F, Taddei D, Grasso L and Pinchera A: Recombinant human thyrotropin-stimulated serum thyroglobulin combined with neck ultrasonography has the highest sensitivity in monitoring differentiated thyroid carcinoma. J Clin Endocrinol Metab 88: 3668-3673, 2003.

24. Torlontano M, Attard M, Crocetti U, Tumino S, Bruno R, Costante G, D'Azzò G, Meringolo D, Ferretti E, Sacco R, et al: Follow-up of low risk patients with papillary thyroid cancer: Role of neck ultrasonography in detecting lymph node metastases. J Clin Endocrinol Metab 89: 3402-3407, 2004.

25. Tuttle RM, Tala H, Shah J, Leboeuf R, Ghossein R, Gonen M, Brokhin M, Omry G, Fagin JA and Shaha A: Estimating risk of recurrence in differentiated thyroid cancer after total thyroidectomy and radioactive iodine remnant ablation: Using response to therapy variables to modify the initial risk estimates predicted by the new American thyroid association staging system. Thyroid 20: 1341-1349, 2010.

26. Leboulleux S, Girard E, Rose M, Travagli JP, Sabbah N, Caillou B, Hartl DM, Lassau N, Baudin E and Schlumberger M: Ultrasound criteria of malignancy for cervical lymph nodes in patients followed up for differentiated thyroid cancer. J Clin Endocrinol Metab 92: 3590-3594, 2007.

27. Pelttari H, Laitinen K, Schalin-Jäntti C and Välimäki MJ: Long-term outcome of 495 TNM stage I or II patients with differentiated thyroid carcinoma followed up with neck ultrasonography and thyroglobulin measurements on T4 treatment. Clin Endocrinol (Oxf) 69: 323-331, 2008.

28. Yoon JH, Kim JY, Moon HJ, Youk JH, Son EJ, Kim EK, Han KH and Kwak JY: Contribution of computed tomography to ultrasound in predicting lateral lymph node metastasis in patients with papillary thyroid carcinoma. Ann Surg Oncol 18: 1734-1741, 2001.

29. Nixon IJ, Ganly I, Patel SG, Palmer FL, Whitcher MM, Tuttle RM, Shaha A and Shah JP: Thyroid lobectomy for treatment of well differentiated intrathyroid malignancy. Surgery 151: 571-579, 2012.

30. Durante C, Attard M, Torlontano M, Ronga G, Monzani F, Costante G, Ferdeghini M, Tumino S, Meringolo D, Bruno R, et al: Identification and optimal postsurgical follow-up of patients with very low-risk papillary thyroid microcarcinomas. J Clin Endocrinol Metab 95: 4882-4888, 2010. 
31. Kim MH, Bae JS, Lim DJ, Lee H, Jeon SR, Park GS and Jung CK: Quantification of BRAF V600E alleles predicts papillary thyroid cancer progression. Endocr Relat Cancer 21: 891-902, 2014.

32. Shin DY, Kim KJ, Chang S, Kim H, Hwang S, Kim W, Bae J, Park S, Kang SW, Chung WY and Lee EJ: Follicular variant of papillary thyroid carcinoma with B-type Raf(V600E) showing higher frequency of suspicious sonographic features and multifocality. Head Neck 37: 1590-1595, 2015.

33. Wada N, Duh QY, Sugino K, Iwasaki H, Kameyama K, Mimura T, Ito K, Takami H and Takanashi Y: Lymph node metastasis from 259 papillary thyroid microcarcinomas: Frequency, pattern of occurrence and recurrence, and optimal strategy for neck dissection. Ann Surg 237: 399-407, 2003

34. Li F, Chen G, Sheng C, Gusdon AM, Huang Y, Lv Z, Xu H, Xing $M$ and Qu S: BRAFV600E mutation in papillary thyroid microcarcinoma: A meta-analysis. Endocr Relat Cancer 22: 159-168, 2015.

35. Guo L, Ma YQ, Yao Y, Wu M, Deng ZH, Zhu FW, Luo YK and Tang J: Role of ultrasonographic features and quantified BRAFV600E mutation in lymph node metastasis in Chinese patients with papillary thyroid carcinoma. Sci Rep 9: 75, 2019.

36. Xing M, Alzahrani AS, Carson KA, Viola D, Elisei R, Bendlova B, Yip L, Mian C, Vianello F, Tuttle RM, et al: Association between BRAF V600E mutation and mortality in patients with papillary thyroid cancer. JAMA 309: 1493-1501, 2013.
37. Lin KL, Wang OC, Zhang XH, Dai XX, Hu XQ and Qu JM: The BRAF mutation is predictive of aggressive clinicopathological characteristics in papillary thyroid microcarcinoma. Ann Surg Oncol 17: 3294-3300, 2010.

38. Miccoli P and Basolo F: BRAF mutation status in papillary thyroid carcinoma: Significance for surgical strategy. Langenbecks Arch Surg 399: 225-228, 2014.

39. Durante C, Puxeddu E, Ferretti E, Morisi R, Moretti S, Bruno R, Barbi F, Avenia N, Scipioni A, Verrienti A, et al: BRAF mutations in papillary thyroid carcinomas inhibit genes involved in iodine metabolism. J Clin Endocrinol Metab 92: 2840-2843, 2007.

40. Zafon C, Baena JA, Castellví J, Obiols G, Monroy G and Mesa J: Differences in the form of presentation between papillary microcarcinomas and papillary carcinomas of larger size. J Thyroid Res 2011: 639156, 2010.

41. Huang K, Gao N, Zhai Q, Bian D, Wang D and Wang X: The anteroposterior diameter of nodules in the risk assessment of papillary thyroid microcarcinoma. Medicine (Baltimore) 97: e9712, 2018. 